\title{
Erratum: High-Fidelity Bidirectional Nuclear Qubit Initialization in SiC [Phys. Rev. Lett. 117, 220503 (2016)]
}

\author{
Viktor Ivády, Paul V. Klimov, Kevin C. Miao, Abram L. Falk, David J. Christle, Krisztián Szász, \\ Igor A. Abrikosov, David D. Awschalom, and Adam Gali \\ (Received 25 May 2017; published 23 June 2017)
}

DOI: 10.1103/PhysRevLett.118.259903

A sign error was found in the definition of the spin Hamiltonian (1) at the nuclear Zeeman term [third term on the right-hand side of Eq. (1)]. The correct form reads

$$
\hat{H}_{\text {spin }}=D\left(\hat{S}_{z}^{2}-\frac{2}{3}\right)+g_{e} \mu_{B} B_{z} \hat{S}_{z}-g_{N} \mu_{N} B_{z} \hat{I}_{z}+\hat{\mathbf{S}}^{T} \mathbf{A} \hat{\mathbf{I}} .
$$

The sign error is present in Eq. (6) as well. The correct expression for $B_{\mathrm{LAC}-c_{ \pm}}$is

$$
B_{\mathrm{LAC}-c_{ \pm}}=\frac{D \mp \frac{A_{z}}{2}}{g_{e} \mu_{B} \pm g_{N} \mu_{N}} .
$$

Because of the incorrect sign of the nuclear Zeeman effect, Fig. 2 should be revised. The updated figure, showing the results of the new simulations, is provided in Fig. 1.

The committed error does not effect our statements and conclusion.

(a)

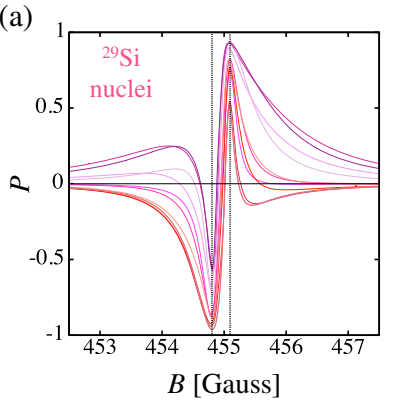

(b)

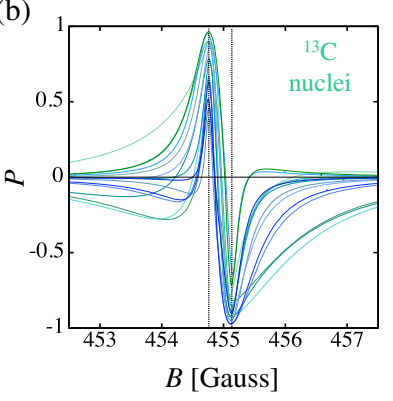

(c)

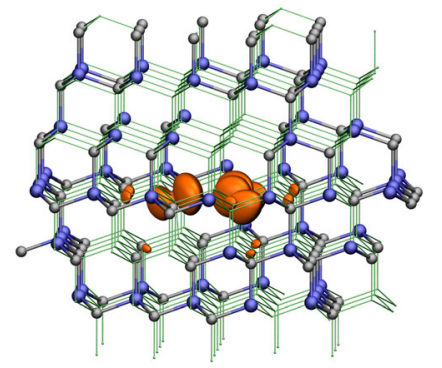

FIG. 1. Magnetic field dependence of the DNP of (a) ${ }^{29} \mathrm{Si}$ and (b) ${ }^{13} \mathrm{C}$ nuclei at different (c) weakly coupled neighboring sites around the $h h$ divacancy in $6 \mathrm{H}-\mathrm{SiC}$. (c) Weakly coupled ${ }^{13} \mathrm{C}$ and ${ }^{29} \mathrm{Si}$ sites around a $h h$ divacancy in $6 \mathrm{H}-\mathrm{SiC}$ which exhibit well-developed peak and dip in their polarization curve. The orange lobes show the spin density of the divacancy that localized on the silicon vacancy site. The greenish wire frame shows the domain around the divacancy, in which the DNP calculations are carried out. Those Si and C sites that show "peak and dip" polarization curve are represented by gray and blue balls, respectively. These sites are situated on an approximately spherical shell around the silicon vacancy site. 